\title{
ANÁLISE DE INCLUSÕES EM AÇO COM FAIXAS DE ALUMÍNIO E ENXOFRE PARA APLICAÇÃO AUTOMOTIVA*
}

\author{
Ayumi Yoshioka ${ }^{1}$ \\ Vinicius Cardoso da Rocha \\ Wagner Viana Bielefeldt ${ }^{3}$
}

\section{Resumo}

Os componentes automotivos em geral devem atender a especificações rigorosas de propriedades mecânicas, requisitos de limpeza inclusionária, entre outros. Enxofre e alumínio são dois elementos adicionados ao banho líquido, que têm papel importante na obtenção destas propriedades, mas que dificultam o processo de produção por lingotamento contínuo por favorecer a formação de partículas sólidas de alto ponto de fusão, que podem prejudicar a limpeza inclusionária e provocar a obstrução das válvulas de lingotamento. $\mathrm{O}$ objetivo do presente trabalho é analisar e classificar as inclusões de corridas do aço DIN 38MnSiVS6 em escala industrial e avaliar estes resultados em conjunto com os dados de processo de aciaria e com os resultados de simulação termodinâmica utilizando o software FactSage 6.4. Foi observado que as corridas apresentaram tanto sulfetos de cálcio quanto espinélios, mas as primeiras, associadas a uma fração líquida menor na escória, tiveram uma correlação mais perceptível com a má lingotabilidade.

Palavras-chave: Análise automatizada de inclusões; Escória; Clogging; Simulação termodinâmica.

\section{INCLUSION ANALYSIS IN STEEL WITH ALUMINUM AND SULPHUR FOR AUTOMOTIVE APPLICATIONS}

\section{Abstract}

The automotive parts usually must meet stringent specification of mechanical properties, cleanliness requirements, dimensional accuracy and high surface quality. Sulfur and aluminum are two elements added to the liquid bath, which play an important role in achieving these properties, but that hinder the production process by continuous casting because these two elements form solid particles with high melting point that can harm the inclusion cleanliness and clog the submerged entry nozzle. The objective of this study is to analyze and classify the inclusions of industrial scale heats of DIN 38MnSiVS6 steel grade and evaluate these results in conjunction with steelmaking data, and with the results of thermodynamic simulation using FactSage 6.4 software. It was observed both calcium sulfides and spinels, but the first one, associated with a lower liquid fraction in the slag, have a more noticeable correlation with poor castability.

Keywords: Automated inclusion analysis; Slag; Clogging; Computational thermodynamics.

1 Eng. Metalúrgico, mestranda, Assessora Técnica/P\&D, Tecnologia de Aços Especiais/P\&D, Gerdau Aços Especiais, Charqueadas, RS, Brasil.

2 Eng. Metalúrgico, mestrando, Laboratório de Siderurgia (LaSid), Depto. de Metalurgia, PPGE3M, UFRGS, Porto Alegre, RS, Brasil.

3 Eng. Metalúrgico, Prof. Dr., Laboratório de Siderurgia (LaSid), Depto. de Metalurgia, PPGE3M, UFRGS, Porto Alegre, RS, Brasil. 


\section{INTRODUÇÃO}

Uma das aplicações do aço DIN 38MnSiVS6, microligado ao manganês - vanádio é o cubo de roda, que funciona como suporte do disco de freio e também serve para transmitir o torque da junta homocinética para as rodas do veículo, dando movimento para o mesmo. Nele estão fixados os parafusos de roda e o rolamento de roda. Uma etapa importante de fabricação deste tipo de peça automotiva é o processo de usinagem após o forjamento, a fim de conferir precisão dimensional e melhorar o acabamento superficial. Além disso, para que se obtenha uma microestrutura homogênea após o resfriamento controlado, é importante um refino de grão austenítico adequado. A adição de enxofre (S) e alumínio (Al) apresentam uma função importante para estes fins. O primeiro deve formar inclusões deformáveis e de baixa temperatura de fusão de sulfetos de manganês, que favorecem a quebra dos cavacos durante a usinagem. Já o segundo, associado a teores adequados de nitrogênio, atua como controlador do crescimento de grão austenítico.

Por outro lado, pelo ponto de vista de fabricação do aço em aciarias com processo de solidificação por lingotamento contínuo, a presença destes dois elementos é conhecida por provocar a formação de inclusões que podem levar à obstrução parcial ou total das válvulas de lingotamento, dando origem ao fenômeno conhecido como clogging [1, 2]. Em geral, a sequência de processo para aços contendo Al e S é: desoxidação durante o vazamento de aço do forno elétrico a arco através de ligas (com ou sem alumínio) dispostas no fundo da panela $\rightarrow$ refino secundário (fornopanela e desgaseificação) $\rightarrow$ adição de fios de alumínio, cálcio-silício e enxofre $\rightarrow$ flotação $\rightarrow$ lingotamento contínuo. Nesta sequência, a adição de Al pode provocar a formação de alumina sólida $\left(\mathrm{Al}_{2} \mathrm{O}_{3}\right)$, que deve ser transformada, através da reação com o cálcio-silício, em inclusões líquidas complexas de cálcio-aluminatos (xCaO$\mathrm{yAl}_{2} \mathrm{O}_{3}$ ) que possam flotar para a escória. Nesta mesma etapa, a formação de sulfeto de cálcio (CaS) deve ser controlada após a adição de $S$, por se tratar de um tipo de inclusão igualmente conhecido por provocar clogging [1, 2].

Em um estudo prévio de simulação termodinâmica na planta industrial de produção de aços especiais onde este trabalho foi desenvolvido, comprovou-se que a janela de lingotabilidade de aços com teores de S e Al similares aos do DIN 38MnSiVS6 (S mínimo 0,040\% e Al mínimo 0,010\%) é bastante restrita [3] (Figura 1).

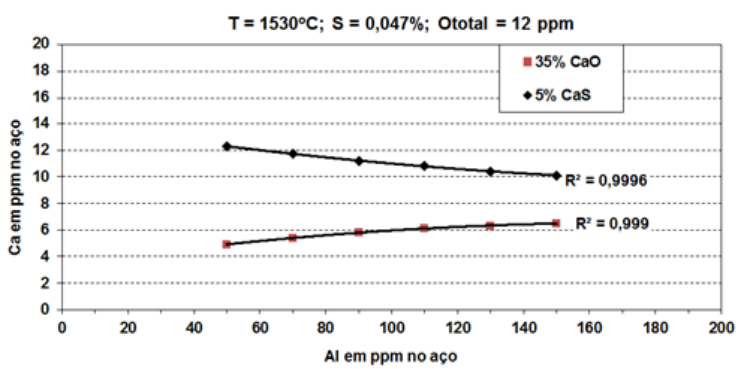

Figura 1. Janela de lingotabilidade.

O presente trabalho visa analisar as inclusões formadas em corridas de produção industrial e correlacionar os resultados com a composição química do aço e da escória com os parâmetros de processo de refino e lingotamento, e com as frações sólida, líquida e viscosidade efetiva da escória de cada corrida, obtidas por simulação termodinâmica. 


\section{MATERIAIS E MÉTODOS}

\subsection{Análise de inclusões do distribuidor}

Amostras de aço líquido de DIN 38MnSiVS6 de quatro corridas foram retiradas do distribuidor na metade do processo de lingotamento das corridas, após aproximadamente 30 minutos da abertura da panela, através de amostrador do tipo lollipop do fabricante Haraeus Electro-Nite, modelo Samp-O-Line.

As amostras foram preparadas por lixamento e polimento metalográfico.

Uma área de aproximadamente $80 \mathrm{~mm}^{2}$ foi analisada em microscópio eletrônico de varredura, acoplado a um espectrômetro de energia dispersiva (MEV/EDS) com um sistema automatizado de análise e classificação de inclusões, do fabricante $\mathrm{FEl}$, modelo Aspex Explorer.

Os equipamentos de análise automatizada de inclusões fornecem dados para um grande número de inclusões, tais como tamanho, composição, distribuição e classificação de inclusões. Entretanto, a amostragem e a área analisada devem ser adequadas para cada tipo de processo produtivo e requisitos de limpeza do aço. Uma forma interessante de interpretação dos resultados é através do uso de diagramas ternários combinados, no qual são plotados os pontos mais representativos de acordo com os três elementos encontrados em maior abundância [4].

Neste trabalho, os resultados obtidos foram processados $e$ as inclusões selecionadas foram plotadas em uma combinação de dois diagramas ternários: sistemas $\mathrm{CaO}-\mathrm{Al}_{2} \mathrm{O}_{3}-\mathrm{MgO}$ (CAM) e Ca-Al-S (CAS). Esta combinação de diagramas ternários é capaz de representar os principais tipos de inclusões sólidas que podem se formar nos aços com altos teores de enxofre e alumínio. Os tipos de inclusões que se pretende identificar no ternário CAM são: alumina $\left(\mathrm{Al}_{2} \mathrm{O}_{3}\right)$, cálcio-aluminatos $\left(\mathrm{xCaO}-\mathrm{yAl}_{2} \mathrm{O}_{3}\right)$ e espinélio $\left(\mathrm{Al}_{2} \mathrm{O}_{3}-\mathrm{MgO}\right)$. No diagrama CAS espera-se verificar se as inclusões do tipo sulfetos de cálcio (CaS) encontradas estão posicionadas nas regiões de inclusões líquidas ou sólidas a $1600^{\circ} \mathrm{C}$. Para o primeiro sistema, os teores elementares foram convertidos em óxidos e depois normalizados. Para o segundo, foi calculada a fração molar e os resultados foram normalizados, sem a conversão para compostos. A seleção das partículas para cada tipo de ternário foi feita de acordo com a Tabela 1. As regras de classificação representam os percentuais em massa dos elementos indicados; a regra 1 foi aplicada para selecionar os três elementos mais representativos para cada diagrama e as demais regras foram aplicadas para excluir desta avaliação de resultados os sulfetos de manganês e as inclusões com alto teor de sílica, por não serem o foco de estudo deste trabalho.

Tabela 1. Regras de classificação por tipo de ternário

\begin{tabular}{lcccc}
\hline Sistema/ Regra & $\mathbf{1}$ & $\mathbf{2}$ & $\mathbf{3}$ & $\mathbf{4}$ \\
\hline $\mathrm{CaO}-\mathrm{Al}_{2} \mathrm{O}_{3}-\mathrm{MgO}$ & $(\mathrm{Ca}+\mathrm{Mg}+\mathrm{Al})$ & $\mathrm{S}<10$ & $\mathrm{Si}<10$ & $\mathrm{Mn}<10$ \\
& $>=50$ & & & \\
\hline $\mathrm{Ca}-\mathrm{Al}-\mathrm{S}$ & $(\mathrm{Ca}+\mathrm{S}+\mathrm{Al})$ & $\mathrm{Si}<10$ & $\mathrm{Mn}<10$ & \\
& $>=80$ & & & \\
\hline
\end{tabular}

Os pontos foram então sobrepostos aos diagramas disponíveis na literatura: $\mathrm{CaO}$ $\mathrm{Al}_{2} \mathrm{O}_{3}-\mathrm{MgO}$ [5] e Ca-Al-S [6] (Figura 2). O diagrama $\mathrm{CaO}-\mathrm{Al}_{2} \mathrm{O}_{3}-\mathrm{MgO}$ apresenta as isotermas, no qual se destacam, na linha binária $\mathrm{CaO}-\mathrm{Al}_{2} \mathrm{O}_{3}$, o cálcio-aluminato $12 \mathrm{CaO} \cdot 7 \mathrm{Al}_{2} \mathrm{O}_{3}(\mathrm{C} 12 \mathrm{~A} 7)$, que é o composto de menor temperatura de fusão e, na 
linha binária $\mathrm{Al}_{2} \mathrm{O}_{3} \cdot \mathrm{MgO}$, o espinélio $\mathrm{Al}_{2} \mathrm{O}_{3} \cdot \mathrm{MgO}$. No diagrama Ca-Al-S [6], quando não há enxofre na inclusão, o mesmo ponto de formação do C12A7 é representado na linha binária Ca-Al no ponto com fração molar de 0,46 de cálcio e, na linha binária Ca-S, o ponto com fração molar de 0,50 de enxofre representa a formação de sulfeto de cálcio sólida a $1600^{\circ} \mathrm{C}$. As demais regiões deste diagrama são compostas de inclusões complexas semissólidas a $1600^{\circ} \mathrm{C}$. Assim, a região indicada na cor cinza na Figura 2 representa a região de inclusões líquidas a $1600^{\circ} \mathrm{C}$.

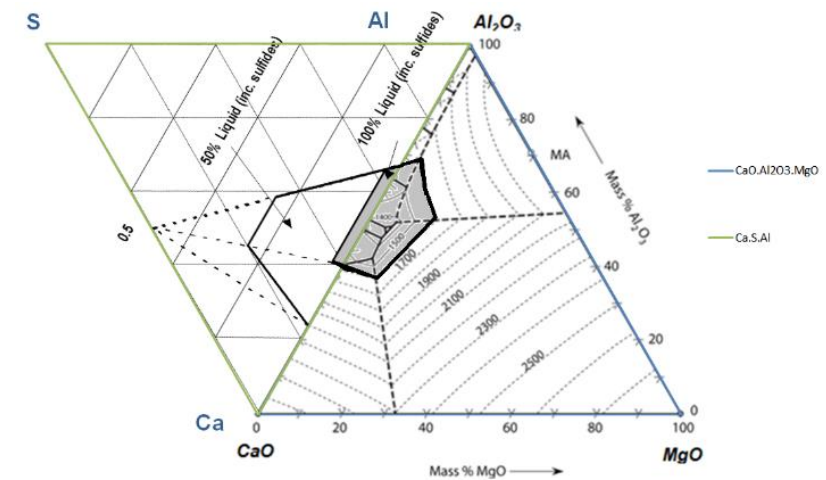

Figura 2. Sobreposição nos sistemas $\mathrm{CaO}-\mathrm{Al}_{2} \mathrm{O}_{3}-\mathrm{MgO}$ (CAM) e Ca-Al-S (CAS) da literatura.

Outro método utilizado para classificar as inclusões foi a quantificação das inclusões em número de inclusões por milímetros quadrados distribuídas por tamanho $(\mu \mathrm{m})$ e por tipo de inclusão: sulfeto de cálcio (CaS) ou espinélio $\left(\mathrm{MgO}-\mathrm{Al}_{2} \mathrm{O}_{3}\right)$, que são os tipos mais comuns de inclusões encontradas nos depósitos de obstrução das válvulas [1,2]. Foram selecionadas as mesmas inclusões do método anterior e foram aplicadas regras adicionais pré-definidas pelo software de classificação do $\mathrm{MEV/EDS}$. Estas regras, estabelecidas de modo a selecionar somente aquelas que representam as inclusões sólidas a $1600^{\circ} \mathrm{C}$, são apresentadas na Tabela 2 , onde os elementos estão indicados em percentual em massa.

Tabela 2. Regras de classificação por tipo de inclusão

\begin{tabular}{lcc}
\hline Tipo/ Regra & $\mathbf{1}$ & $\mathbf{2}$ \\
\hline $\mathrm{CaS}$ & $\mathrm{Ca}>=20$ & $\mathrm{~S}>=20$ \\
\hline $\mathrm{MgO}-\mathrm{Al}_{2} \mathrm{O}_{3}$ & $\mathrm{Al}>=30$ & $\mathrm{Mg} /(\mathrm{Al}+\mathrm{Mg}+\mathrm{Ca})>=0,10$ \\
\hline
\end{tabular}

\subsection{Análise de composição química}

A composição química das amostras de aço das etapas de refino, desgaseificação e lingotamento foram obtidas nos seguintes equipamentos:

- Espectrômetro de emissão ótica, do fabricante ARL, modelo 4460;

- Determinador simultâneo de Carbono e Enxofre da fabricante LECO, modelo CS-444LS;

- Determinador simultâneo de Nitrogênio e Oxigênio da fabricante LECO, modelo TC-436.

A composição química das amostras de escória da etapa de refino e desgaseificação foi obtida via fluorescência de raios-x do fabricante Philips, modelo PW2600.

\subsection{Avaliação dos parâmetros de processo}

Foram avaliados os seguintes parâmetros de processo de refino e lingotamento: 
- Temperaturas de processamento nas etapas de refino secundário (fornopanela e desgaseificação) e lingotamento contínuo.

- Quantidade de fios de alumínio, cálcio silício e enxofre.

- Tempo de flotação após a adição dos fios.

- Variação da altura do tampão entre 5 e 55_min após o início do lingotamento.

\subsection{Simulação termodinâmica}

Foi utilizado o software comercial FactSage 6.4, módulos Equilib e Viscosity, do Laboratório de Siderurgia da Universidade Federal do Rio Grande do Sul. No módulo Equilib, os bancos de dados aplicados foram o FACT-FactPS (substâncias puras estequiométricas) e FACT-FToxid (óxidos e enxofre). A partir do emprego destes bancos de dados foram calculadas as frações sólida, líquida e viscosidade efetiva de cada escória para cada corrida.

\section{RESULTADOS E DISCUSSÃO}

\subsection{Composição Química}

A Tabela 3 apresenta o resultado de composição química do aço no distribuidor.

Tabela 3. Composição química do aço no distribuidor (\% massa)

\begin{tabular}{ccccccccccc}
\hline Corrida & $\mathbf{C}$ & $\mathbf{S i}$ & $\mathbf{M n}$ & $\mathbf{C r}$ & $\mathbf{N 2}$ & $\mathbf{T i}$ & $\mathbf{V}$ & $\mathbf{S}$ & $\mathbf{A l}$ & $\mathbf{C a}$ \\
\hline C1 & 0,37 & 0,67 & 1,34 & 0,14 & 0,013 & 0,017 & 0,10 & 0,049 & 0,012 & 0,0008 \\
\hline C2 & 0,37 & 0,62 & 1,31 & 0,14 & 0,013 & 0,017 & 0,09 & 0,046 & 0,014 & 0,0011 \\
\hline C3 & 0,37 & 0,62 & 1,32 & 0,15 & 0,013 & 0,016 & 0,09 & 0,043 & 0,013 & 0,0006 \\
\hline C4 & 0,36 & 0,65 & 1,33 & 0,14 & 0,012 & 0,016 & 0,10 & 0,040 & 0,014 & 0,0012 \\
\hline
\end{tabular}

O aço no distribuidor apresenta a composição química final de cada corrida, após todas as adições de fios e remoção de inclusões. Verificou-se pouca dispersão no alumínio; as corridas C1 e C2 apresentaram teores um pouco mais elevados de enxofre em relação às corridas C3 e C4; e o percentual de cálcio das corridas C2 e $\mathrm{C} 4$ foi um pouco maior em relação às outras duas corridas.

O posicionamento das corridas na Janela de lingotabilidade é ilustrado na Figura 3. Verifica-se que para o teor de alumínio indicado, há um estreitamento da faixa ideal de cálcio para que sejam obtidas inclusões líquidas. A corrida C1 encontra-se dentro da janela líquida e as demais corridas estão muito próximas, mas a C3 está em uma região com início de precipitação de cálcio-aluminatos e as corridas C2 e C4, de sulfeto de cálcio.

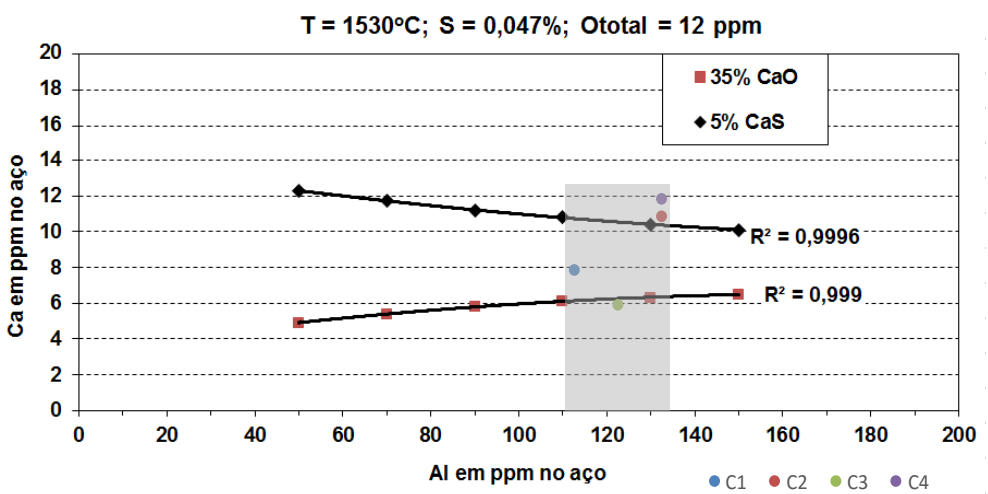

Figura 3. Posicionamento das corridas na janela de lingotabilidade. 
A Tabela 4 abaixo mostra os principais compostos da escória de desgaseificador.

Tabela 4. Composição química da escória no desgaseificador (\% massa)

\begin{tabular}{lccccc}
\hline Corrida & $\mathbf{C a O}$ & $\mathbf{S i O}_{2}$ & $\mathbf{A l}_{2} \mathbf{O}_{3}$ & $\mathbf{M g O}$ & $\mathbf{S}$ \\
\hline $\mathrm{C} 1$ & 45,7 & 22,4 & 12,9 & 16,3 & 0,86 \\
\hline C2 & 43,6 & 22,6 & 14,9 & 15,9 & 0,68 \\
\hline C3 & 49,0 & 20,8 & 15,4 & 11,3 & 1,18 \\
\hline C4 & 44,2 & 20,6 & 14,4 & 17,0 & 1,11 \\
\hline
\end{tabular}

A escória do desgaseificador tem a principal função de remoção de inclusões, uma vez que no distribuidor é utilizada uma nova escória sintética para proteção contra reoxidação e manutenção de temperatura. Os valores encontrados estão em conformidade com a dispersão esperada para este tipo de escória.

\subsection{Análise de inclusões}

As inclusões encontradas nas quatro corridas foram representadas conforme combinação de diagramas ternários (Figura 4).
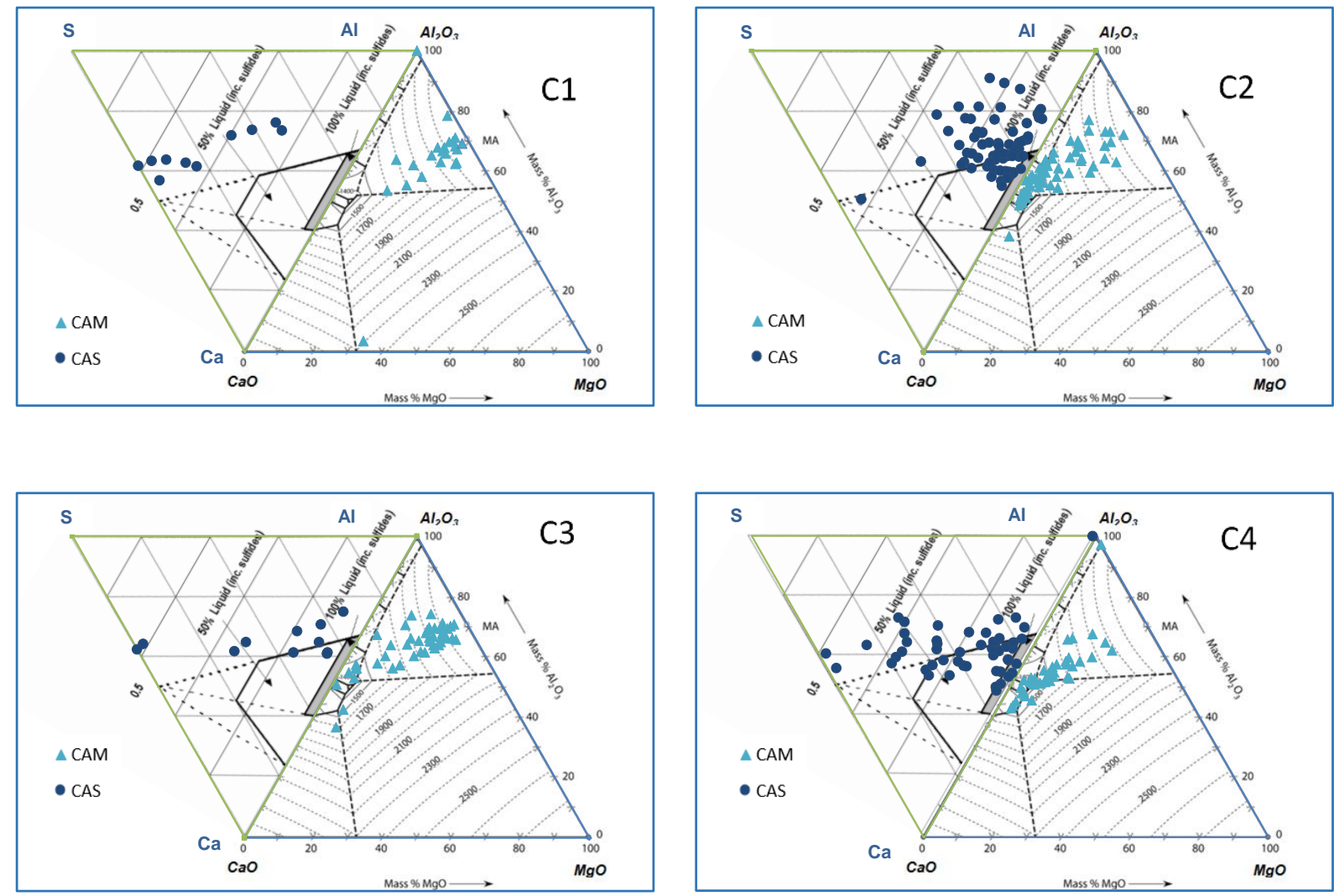

Figura 4. Inclusões nos sistemas $\mathrm{CaO}-\mathrm{Al}_{2} \mathrm{O}_{3}-\mathrm{MgO}(\mathrm{CAM})$ e $\mathrm{Ca}-\mathrm{Al}-\mathrm{S}(\mathrm{CAS})$.

Analisando estes diagramas:

- No diagrama CAM, a corrida C1 apresenta muitas inclusões no campo do espinélio (MA) e praticamente nenhuma no campo de cálcio-aluminatos líquidos (CA). As inclusões no ternário CAS estão igualmente fora do campo líquido a $1600^{\circ} \mathrm{C}$. 
- As corridas C2 e C4 apresentam muitas inclusões no campo de inclusões líquidas de CA em ambos os diagramas, mas há uma grande quantidade de inclusões fora do campo líquido a $1600^{\circ} \mathrm{C}$ no ternário CAS.

- A corrida C3 apresenta poucas inclusões no campo líquido de CA e algumas se aproximando do ponto MA. Há poucas ocorrências no diagrama CAS.

Outra forma de representação utilizada foi a distribuição por tamanho e por tipo de inclusão (Figura 5).
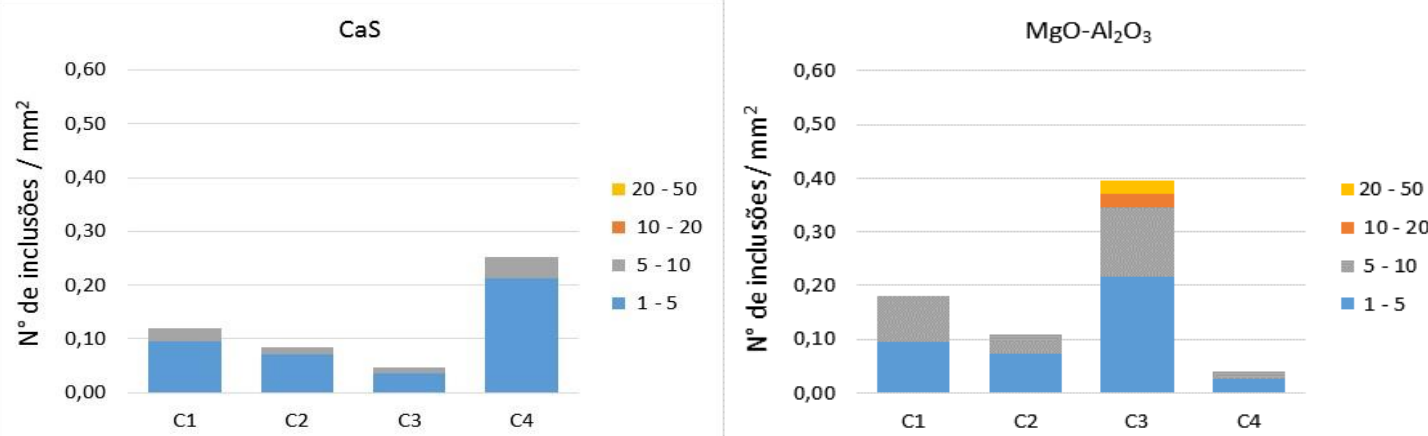

Figura 5. Número de inclusões por $\mathrm{mm}^{2}$ distribuídas por tamanho $(\mu \mathrm{m})$ e por tipo de inclusão CaS e $\mathrm{MgO}-\mathrm{Al}_{2} \mathrm{O}_{3}$.

Esta representação complementa as informações dos diagramas ternários da Figura 4 , pois nesta é possível realizar um comparativo quantitativo entre as corridas. Neste trabalho são abordados somente os tipos $\mathrm{CaS}$ e o $\mathrm{MgO}-\mathrm{Al}_{2} \mathrm{O}_{3}$, pois estes são os tipos mais comuns de se encontrar nos depósitos das válvulas.

A densidade de inclusões do tipo sulfeto de cálcio da corrida C4 foi a maior de todas, seguida pelas corridas C1, C2 e C3. Já em relação aos espinélios, a corrida C3 apresentou a maior densidade, seguida pelas corridas C1, C2 e C4. Nota-se que a maioria das inclusões encontradas está na faixa de 1 a $5 \mu \mathrm{m}$, e as ocorrências acima de $10 \mu \mathrm{m}$ foram observadas somente na corrida C3 para o tipo espinélios.

\subsection{Simulações termodinâmicas}

Os resultados a seguir foram obtidos a partir das composições normalizadas das escórias: $\mathrm{CaO}, \mathrm{SiO}_{2}, \mathrm{MgO}_{\text {e } \mathrm{Al}_{2} \mathrm{O}_{3}}$ (Tabela 5) a uma temperatura de $1630^{\circ} \mathrm{C}$, que foi a temperatura média da etapa de desgaseificação, pois é nessa etapa que ocorrem os mecanismos principais de remoção de inclusões: flotação, separação e dissolução [7].

Tabela 5. Fração sólida, líquida e viscosidade efetiva da escória no desgaseificador a $1630^{\circ} \mathrm{C}$

\begin{tabular}{cccc}
\hline Corrida & $\begin{array}{c}\text { Fração } \\
\text { sólida }\end{array}$ & $\begin{array}{c}\text { Fração } \\
\text { líquida }\end{array}$ & $\begin{array}{c}\text { Viscosidade } \\
\text { efetiva (Pa.s) }\end{array}$ \\
\hline C1 & 0,15 & 0,85 & 0,110 \\
\hline C2 & 0,07 & 0,93 & 0,092 \\
\hline C3 & 0,05 & 0,95 & 0,078 \\
\hline C4 & 0,10 & 0,90 & 0,094 \\
\hline
\end{tabular}

Destaca-se que a fração líquida das corridas C2 e C3 apresentaram-se como as maiores, de 0,93 e 0,95, contra 0,85 e 0,90 para as corridas $\mathrm{C} 1$ e C4, respectivamente. 


\subsection{Correlação da lingotabilidade com as inclusões encontradas, as simulações termodinâmicas e os demais parâmetros de processo}

Conforme observações do comportamento de corridas anteriores desta qualidade de aço em estudo, ocorre um aumento da altura do tampão entre o final e o início do lingotamento, o que indica que está ocorrendo a obstrução das válvulas. A variação observada entre 5 e 55min após o início do lingotamento nas corridas selecionadas é apresentada a seguir (Figura 6).

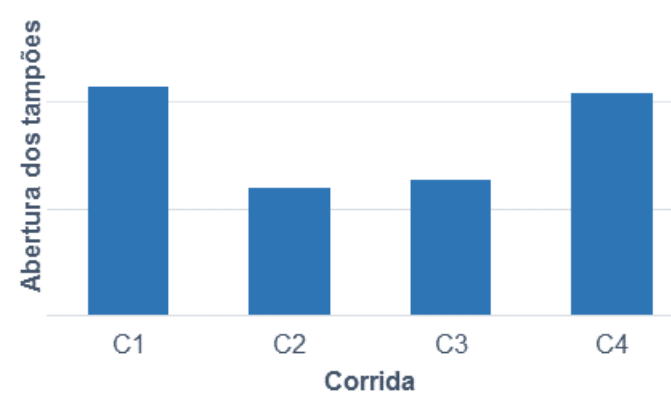

Figura 6. Variação da altura do tampão.

As piores condições de lingotabilidade foram observadas nas corridas $\mathrm{C} 1$ e $\mathrm{C} 4$. As principais correlações destas duas corridas com os tipos de inclusões encontradas foi que estas duas apresentaram a maior densidade de inclusões $\left(\mathrm{N} / \mathrm{mm}^{2}\right)$ do tipo CaS (Figura 5).

A corrida $\mathrm{C} 3$ apresentou um maior número de espinélios, mas pela posição das inclusões no sistema CMA (Figura 4) as inclusões desta corrida contêm um certo teor de cálcio, mostrando que não se tratam de inclusões de espinélio puras. Este pode ser o motivo de não ter sido observada uma abertura acentuada do tampão.

Os resultados de simulação termodinâmica (Tabela 5) revelaram menores frações líquidas nas corridas $\mathrm{C} 1$ e $\mathrm{C} 4$ em relação às corridas $\mathrm{C} 2$ e C3. Esta informação é coerente com a variação da altura do tampão. Para comprovar a correlação entre a lingotabilidade e a fração líquida, foram analisados os dados de dez corridas adicionais deste mesmo aço (Figura 7).

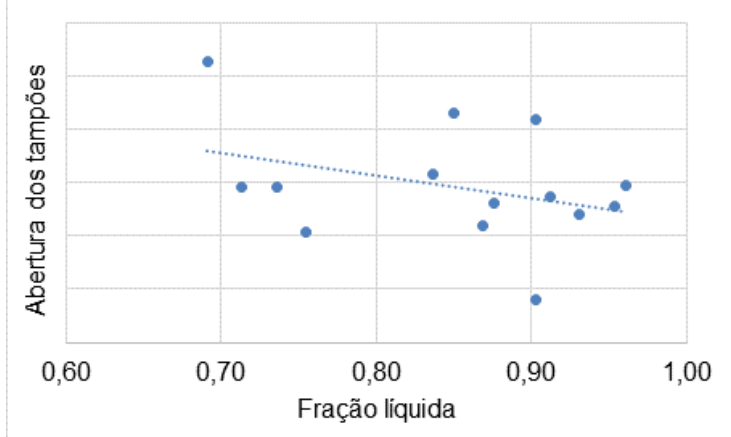

Figura 7: Abertura dos tampões e fração líquida.

O gráfico apresentado na Figura 7 confirma a ocorrência de menor abertura dos tampões para escórias com frações líquidas maiores. Isto pode ser explicado pelo fato de esta escória ter uma maior capacidade de remover inclusões em relação às escórias com fração sólida maior. 
Com relação aos demais parâmetros mencionados no item 2.3, tais como temperaturas, quantidades de fios e tempos de flotação, não foram observadas diferenças significativas entre as quatro corridas e por isso não foram abordadas nesta discussão de resultados.

\section{CONCLUSÕES}

As análises de inclusões das corridas avaliadas evidenciaram a presença tanto de sulfetos de cálcio quanto de espinélios. O uso de diagramas ternários combinados facilita a visualização dos resultados.

As piores condições de lingotabilidade foram verificadas nas corridas com uma densidade maior de inclusões do tipo sulfeto de cálcio nas amostras de aço e que ao mesmo tempo apresentaram uma escória com menor fração líquida.

A metodologia de avaliação de inclusões apresentada neste trabalho pode ser utilizada para avaliar o perfil inclusionário de outros tipos de aço. As conclusões deste estudo podem ser utilizadas para a melhoria contínua do processo de produção e o conhecimento adquirido pode ser ampliado para o aprimoramento da qualidade dos demais produtos produzidos pela empresa.

\section{REFERÊNCIAS}

1 Bielefeldt WV. Tratamento de inclusões não metálicas com cálcio nos aços SAE 1141 e SAE 8620. Porto Alegre. Tese [Doutorado em Engenharia] - Programa de Pós-Graduação em Engenharia de Minas, Metalúrgica e de Materiais - UFRGS; 2009.

2 Guo YT, He SP, Chen GJ, Zhao S, Li ZR, Wu T et al. Improvement in castability of Al containing resulphurised free machining steel. Ironmaking and Steelmaking. 2014; 41(10): 738-747.

3 Universidade Federal do Rio Grande do Sul, Escola de Engenharia, Centro de Tecnologia, Laboratório de Siderurgia. Desenvolvimento de janelas de lingotabilidade para aços críticos. 2013. Relatório Técnico RTE-2007.

4 Harris M, Adaba O, Lekakh S, O'Malley R, Richards L. Improved Methodology for Automated SEM/EDS Non-Metallic Inclusion Analysis of Mini-Mill and Foundry Steels. In: AISTech 2015 Proceedings. 2015: 3315-3325.

$5 \quad$ Slag Atlas. 2. ed. Düsseldorf: Verlag Stahleisen GmbH; 1995.

6 Ahlborg K, Fruehan RJ, Potter MS, Badger SR, Casuccio GS. Inclusions in AluminumKilled Steel with Varying Calcium Additions. In: ISSTech 2003 Conference Proceedings. 2003: 177-194.

7 Abraham S, Bodnar R, Raines J. Inclusion engineering and the metallurgy of calcium treatment. In: AISTech 2013 Proceedings. 2013: 1243-1257. 